\title{
Leukocyte Populations are Associated with Heart Rate Variability After a Triathlon
}

\author{
by \\ Germán Hernández Cruz¹, José Naranjo Orellana², Adrián Rosas Taraco³, \\ Blanca Rangel Colmenero ${ }^{1}$
}

The purpose of this study was to analyze cellular immune components and their association with heart rate variability in triathlon athletes. Twelve athletes were included (age $36.41 \pm 5.57$ years, body mass $81.84 \pm 10.97 \mathrm{~kg}$ ) and blood samples were taken one week before, immediately, at 2 and 48 hours, and one week after competition. Total lymphocytes and their subpopulations, neutrophils, basophils, eosinophils and monocytes were analyzed. At the same time, heart rate variability was recorded for 30 minutes using Polar Team2®. A significant difference between lymphocyte subpopulations and heart rate variability was found in the different study periods. A positive correlation was found between total lymphocytes and $r M S S D(r=.736, p<0.05), C D 3+$ and $r M S S D(r=.785, p<0.05)$, and CD4+ and $r M S S D(r=.795, p<0.05)$ at the end of the competition. After one week of competition, a negative correlation was found between eosinophils and MRR, SDNN, pNN50, and rMSSD ( $<<0.01)$; and basophils and MRR, SDNN, $p N N 50$, and $r M S S D(p<0.01)$; while a positive correlation was found between CD19+ (B cells) and $p N N 50(r=.678, p$ $<0.05)$. Our results suggest that it is possible to predict the effect of training with regard to the athlete's performance.

Key words: exercise, lymphocytes, training, competition..

\section{Introduction}

The study of immunology during exercise is based on analyzing the acute and chronic effects of training on the immune system, the inflammatory process, and the effect of exercise on the incidence of upper airway infections (Kakanis et al., 2010). Studies report that exercise has a modulatory function depending on the intensity at which it is practiced, i.e., during moderate physical activity, the immune response improves its function, and conversely, it decreases with high-intensity physical activity (Cordova Sureda et al., 2010; Nieman, 2011; Scharhag, 2005). During intense training or competition, the athlete's immune system response suffers a decrease, which means increased susceptibility to infections and diseases associated with the immune system (Morgado et al., 2012; Nieman, 2011). This event is known as an "open window" (Malm, 2006; Nieman, 2007; Scharhag, 2005).

Therefore, it is important to examine the immune system of athletes during training or competition, since the suspension of training caused by disease, with regard to the immune system, can negatively influence the development of athletic performance (Kakanis et al., 2010). It has been suggested that strenuous exercise leads to temporary immunosuppression, causing athletes to acquire more respiratory infections during periods of intense training and after competition (Levada-Pires et al., 2008; Tossige-

1 - Faculty of Sports Organization, Autonomous University of Nuevo Leon, Mexico.

2- Department of Sport and Informatics, University Pablo of Olavide, Spain.

2- Faculty of Medicine, Autonomous University of Nuevo Leon, Mexico. 
Gomes et al., 2014). This temporary depression of immune function is a result of acute exercise or competition. Acute exercise also results in an increase of leukocytes and neutrophils, which are the first to migrate to the area of injury (de Moura et al., 2012; Tossige-Gomes et al., 2014); however, lymphocyte circulation is reduced (Gleeson, 2007; Park and Park, 2008).

The autonomic nervous system plays an important role in regulating the immune system (Marsland et al., 2007). An imbalance in sympathetic or parasympathetic activity compromises immune regulation and therefore, increases the risk of disease. It is generally assumed that sympathetic control promotes the inflammatory response while parasympathetic control regulates it (Hellard et al., 2011). Analysis of heart rate variability (HRV) provides information on the autonomic nervous system (Buchheit et al., 2011), which can be measured by time-domain variables that result from ECG measurement of normal NN (normal-to-normal) intervals. These NN intervals are statistically and mathematically analyzed to obtain various parameters. MRR indicates the mean of all RR intervals in a time interval, the SDNN index (standard deviation of all $\mathrm{NN}$ intervals measured in a given period) is an independent indicator of frequencies used to define the concept of total variability, and rMSSD is the square root of the mean of the sum of the squared differences of all successive NN intervals. This parameter conveys the short-term variations of the $\mathrm{NN}$ intervals and it is used to observe the influence of the parasympathetic nervous system on the cardiovascular system. The pNN50 measures the percentage of consecutive NN intervals that differ by more than 50 milliseconds; a high value of pNN50 provides valuable information about the high spontaneous variations in the heart rate (Malik et al., 1996).

Through measurement of HRV with a variable time domain, either in terms of training or competition (Bricou et al., 2010; Plews et al., 2013), we obtain information regarding the dominant stimulus; i.e., in appropriate adaptation to workloads parasympathetic activity dominates; conversely, in overall maladaptive situations, a predominance of sympathetic activity occurs (Bricout et al., 2010; Mazon et al., 2013; Plews et al., 2013).
In this study, the effects of competition and recovery during and after a triathlon on leukocytes and heart rate variability, and the correlation between them were examined.

\section{Material and Methods}

\section{Subjects}

Twelve male amateur triathletes (age $36.41 \pm 5.57$ years, body height $179.33 \pm 8.02 \mathrm{~m}$, body mass $81.84 \pm 10.97 \mathrm{~kg}$ ) with at least five years of sports experience and with six regular training sessions per week, were evaluated. The individuals were in good health and were not consuming any medication that could affect the variables analyzed. They participated in the research voluntarily with prior information on the purpose of the study and the conditions in which work would be done. They signed a letter of informed consent with previous approval by the COFEPRIS ethics/bioethics committee of the Autonomous University of Nuevo Leon.

\section{Sampling}

Blood samples for analysis of the immune response were taken as follows: the subject was placed in a prone position, performing asepsis with $70 \%$ ethanol; a superficial forearm vein, preferably the ulnar or cephalic one, was selected taking three milliliters of blood by puncture with a Vacutainer ${ }^{\mathrm{TM}}$ in a heparinized tube.

\section{Cell count}

For white blood cell counts, the number of total lymphocytes, neutrophils, basophils, eosinophils and monocytes were determined by a differential blood count in a fraction of the blood sample in a reference laboratory. The second fraction of heparinized blood was collected for quantification of lymphocyte subpopulations, placing $100 \mu \mathrm{L}$ of venous blood in four different tubes: in the first tube CD3-FITC/CD4-PE antihuman antibody was placed (to detect $\mathrm{T}$ helper cells), in the second tube CD3-FITC/anti-CD8-PE human antibody was placed (to detect cytotoxic $\mathrm{T}$ lymphocytes), in the third tube anti-human CD3FITC/(CD16/CD56)-PE was placed (to identify NK cells), and in the fourth tube anti-human CD3FITC/CD19-PE was placed (to identify $\mathrm{T}$ and $\mathrm{B}$ lymphocytes), and an isotype control. Later, the samples were incubated in the dark for $15 \mathrm{~min}$ at room temperature, after which $1 \mathrm{~mL}$ of erythrocyte lysis solution was added and the samples were incubated for five minutes in 
darkness. The samples were centrifuged at 1200 rpm for $10 \mathrm{~min}$, discarding the supernatant; they were then washed twice with PBS $\mathrm{pH}$ 7.2, centrifuged each time at $1200 \mathrm{rpm} / 10 \mathrm{~min}$, discarding the supernatant. Finally, the samples were resuspended in $500 \mu \mathrm{L}$ of PBS and analyzed on a flow cytometer (BD FACS Calibur, BD Biosciences, Franklin Lakes, NJ) to calculate the amount of each cell in the samples.

Heart Rate Variability. The variables considered for HRV were those of the time domain: pNN50, MRR, SDNN and rMSSD recorded supine for $30 \mathrm{~min}$ with matching blood samples. Polar Team 2 (Polar Electro Inc., Lake Success, NY) was used in the R-R (beat to beat) option with 10 bands (WearLink wind Polar), which were placed on the chest of each athlete. Data analysis was performed with Polar Protrainer Version 5 computer software. Habits and conditions that could alter the HRV results such as: rest, intake of stimulants, food intake prior to measurement and the emotional state were monitored, following the recommendations of the Task Force of the European Society of Cardiology and the North American Society of Pacing and Electrophysiology (Malik et al., 1996).

Protocol

All study subjects participated in a triathlon competition in the Olympic mode. Five blood sample collections plus HRV recordings were performed. The first took place one week before the competition in complete rest, the second at the end of the competition, the third at two hours, the fourth at $48 \mathrm{~h}$ and the fifth after one week of active recovery.

\section{Statistical analysis}

Descriptive statistics were used (mean \pm standard deviation), as well as Spearman correlations, and for contrast of means, the Kruskal-Wallis test was applied, because the data were non-parametric. All statistical tests were performed in SPSS 19 for Windows.

\section{Results}

All subjects completed the study and the descriptive data of the lymphocytes, leukocytes and HRV, are presented in Table 1.

Leukocytes showed a significant difference between all samples $(\mathrm{F}=24.074, p<$ 0.01 ), increasing their level immediately after the competition and continuing with the same trend up to two hours. At 48 hours they recovered their initial values and remained this way after one week. Neutrophils $(F=27.35, p<0.01)$ showed a similar behavior increasing immediately after the competition and after two hours. At 48 hours, they recovered to normal values and stayed at this level one week after the competition. Lymphocytes $(\mathrm{F}=22.962, p<0.01)$ had a contrary behavior, in other words, they decreased at the end of the competition and at two hours and recovered in subsequent samples (Figure 1).

Monocytes $(\mathrm{F}=8.17, p<0.01)$ increased at the end of the competition and even more at two hours, recovering in subsequent samples. Basophils ( $F=3.39, p<0.01)$ also increased at the end of the competition, although decreased at two hours recovering at 48 hours. Eosinophils $(\mathrm{F}=$ 8.313, $p<0.01$ ) showed a decrease immediately after the competition and continued to decline at two hours, returning to baseline values at 48 hours and one week after the competition (Figure 2).

In the lymphocyte subpopulation, only CD3+ $(\mathrm{F}=2.644, p<0.01), \mathrm{CD} 4+(\mathrm{F}=2.162, p<$ $0.05)$, and $\mathrm{CD} 8+(\mathrm{F}=3.77, p<0.01)$ decreased immediately after the competition returning to the reference values at $48 \mathrm{~h}$, staying at this level one week after the competition (Figure 3).

Correlation of immune response with VFC

The correlations between the variables of the immune response and HRV are shown in Tables 2 and 3. The results after the competition and the positive relationship between rMSSD and total lymphocytes $(\mathrm{r}=.736, p<0.05), \mathrm{CD} 3+(\mathrm{r}=$ $.785, p<0.05)$ and CD4 $+(\mathrm{r}=.795, p<0.05)$ are shown in Table 2. The data one week after the competition showed a negative correlation between eosinophils and MRR $(\mathrm{r}=-.823, p<0.01)$, SDNN $(\mathrm{r}=-.870, p<0.01)$, with pNN50 $(\mathrm{r}=-806, p$ $<0.01)$ and $\operatorname{rMSSD}(\mathrm{r}=-828, p<0.01)$; basophils also negatively correlated with MRR $(\mathrm{r}=-.748, p<$ $0.05)$, SDNN $(\mathrm{r}=-.854, p<0.01)$, pNN50 $(\mathrm{r}=-.817$, $p<0.01)$ and rMSSD $(\mathrm{r}=-.903, p<0.01)$; in contrast, CD19+ B lymphocytes were positively correlated with pNN50 $(r=.678, p<0.05)$.

\section{Discussion}

The results of the study show that strenuous physical activity after a triathlon competition induces significant leukocytosis mainly caused by an increase in neutrophils, and 
lymphopenia of $\mathrm{CD} 3+, \mathrm{CD} 8+$ and $\mathrm{CD} 4+$; these variations correlate with rMSSD. Neutrophils are cells that perform phagocytosis and lysis of microorganisms. They are attracted to foci of infection and inflammation, increasing in the bloodstream due to mobilization from the periphery and yellow bone marrow (Levada-Pires et al., 2008; Park and Park, 2008; Santos et al., 2013; Wolach et al., 2005).

\begin{tabular}{|c|c|c|c|c|c|c|}
\hline \multicolumn{7}{|c|}{$\begin{array}{c}\text { Table } \mathbf{1} \\
\text { Descriptive statistics of the variables analyzed. Data expressed as mean } \pm S D .\end{array}$} \\
\hline & $\mathrm{N}$ & $\begin{array}{l}1 \text { week before } \\
\text { competition }\end{array}$ & $\begin{array}{l}\text { At the end of } \\
\text { competition }\end{array}$ & $\begin{array}{c}2 \mathrm{~h} \text { after } \\
\text { competition }\end{array}$ & $\begin{array}{l}48 \mathrm{~h} \text { after } \\
\text { competition }\end{array}$ & $\begin{array}{l}\text { After one week } \\
\text { active recovery }\end{array}$ \\
\hline Leukocytes $(\mathrm{k} / \mu \mathrm{L})$ & 12 & $6.30 \pm 1.21$ & $10.62 \pm 5.05$ & $12.44 \pm 1.90$ & $6.41 \pm 1.39$ & $6.26 \pm 1.34$ \\
\hline Neutrophils $(\mathrm{k} / \mu \mathrm{L})$ & 12 & $3.17 \pm 0.85$ & $8.37 \pm 4.35$ & $10.04 \pm 1.55$ & $3.16 \pm 0.93$ & $2.90 \pm 0.82$ \\
\hline 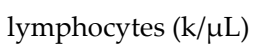 & 12 & $2.21 \pm 0.41$ & $1.49 \pm 0.96$ & $1.49 \pm 0.43$ & $2.38 \pm 0.68$ & $2.35 \pm 0.74$ \\
\hline Monocytes $(\mathrm{k} / \mu \mathrm{L})$ & 12 & $0.64 \pm 0.16$ & $0.64 \pm 0.32$ & $0.84 \pm 0.20$ & $0.57 \pm 0.17$ & $0.68 \pm 0.18$ \\
\hline Eosinophils $(\mathrm{k} / \mu \mathrm{L})$ & 12 & $0.24 \pm 0.10$ & $0.09 \pm 0.09$ & $0.03 \pm 0.03$ & $0.26 \pm 0.09$ & $0.28 \pm 0.13$ \\
\hline Basophils $(\mathrm{k} / \mu \mathrm{L})$ & 12 & $0.04 \pm 0.03$ & $0.04 \pm 0.02$ & $0.03 \pm 0.01$ & $0.04 \pm 0.02$ & $0.04 \pm 0.01$ \\
\hline $\mathrm{CD} 3+\left(\right.$ cells $\left./ \mathrm{mm}^{3}\right)$ & 12 & $1390.55 \pm 374.71$ & $858.84 \pm 561.77$ & $826.59 \pm 280.54$ & $1553.20 \pm 572.10$ & $1489.14 \pm 540.50$ \\
\hline $\mathrm{CD} 4+\left(\right.$ cells $\left./ \mathrm{mm}^{3}\right)$ & 12 & $899.05 \pm 266.21$ & $485.79 \pm 362.14$ & $517.34 \pm 192.67$ & $778.97 \pm 431.89$ & $914.69 \pm 354.41$ \\
\hline $\mathrm{CD} 8+\left(\right.$ cells $\left./ \mathrm{mm}^{3}\right)$ & 12 & $548.96 \pm 175.74$ & $277.09 \pm 121.70$ & $275.66 \pm 99.61$ & $477.10 \pm 142.70$ & $539.63 \pm 204.99$ \\
\hline MRR (ms) & 12 & $1061.46 \pm 111.12$ & $1108.57 \pm 101.32$ & $1064.88 \pm 160.15$ & ND & $1224.13 \pm 125.64$ \\
\hline SDNN (ms) & 12 & $124.32 \pm 43.61$ & $135.57 \pm 26.41$ & $143.84 \pm 57.25$ & ND & $153.12 \pm 48.31$ \\
\hline pNN50 (\%) & 12 & $27.80 \pm 15.97$ & $36.62 \pm 16.54$ & $33.22 \pm 18.71$ & ND & $39.49 \pm 19.70$ \\
\hline rMSSD (ms) & 12 & $40.78 \pm 13.52$ & $46.46 \pm 18.80$ & $48.35 \pm 25.83$ & ND & $57.37 \pm 26.46$ \\
\hline \multicolumn{7}{|c|}{$N D=$ Not determined } \\
\hline
\end{tabular}

Table 2

Correlations in the variables analyzed at the end of the competition

\begin{tabular}{ll}
\multicolumn{1}{c}{ Cell type } & \multicolumn{1}{c}{ rMSSD } \\
\hline Lymphocytes & $.736\left(^{*}\right)$ \\
Lymphocytes T CD3+ & $.785\left(^{*}\right)$ \\
Lymphocytes T CD4+ & $.795\left(^{*}\right)$ \\
\hline
\end{tabular}

*Correlation is significant at $p<0.05$.

Table 3

Correlation in the variables analyzed after one week of active recovery following the competition

\begin{tabular}{|c|c|c|c|c|}
\hline Cell type & MRR & SDNN & pNN50 & rMSSD \\
\hline Eosinophils & $-.823(* *)$ & $-.870\left(^{* *}\right)$ & $-.806\left(^{* *}\right)$ & $-.828\left(^{* *}\right)$ \\
\hline Basophils & $-.748\left(^{*}\right)$ & $-.854\left(^{* *}\right)$ & $-.817\left(^{* *}\right)$ & $\left.-.9033^{* *}\right)$ \\
\hline
\end{tabular}




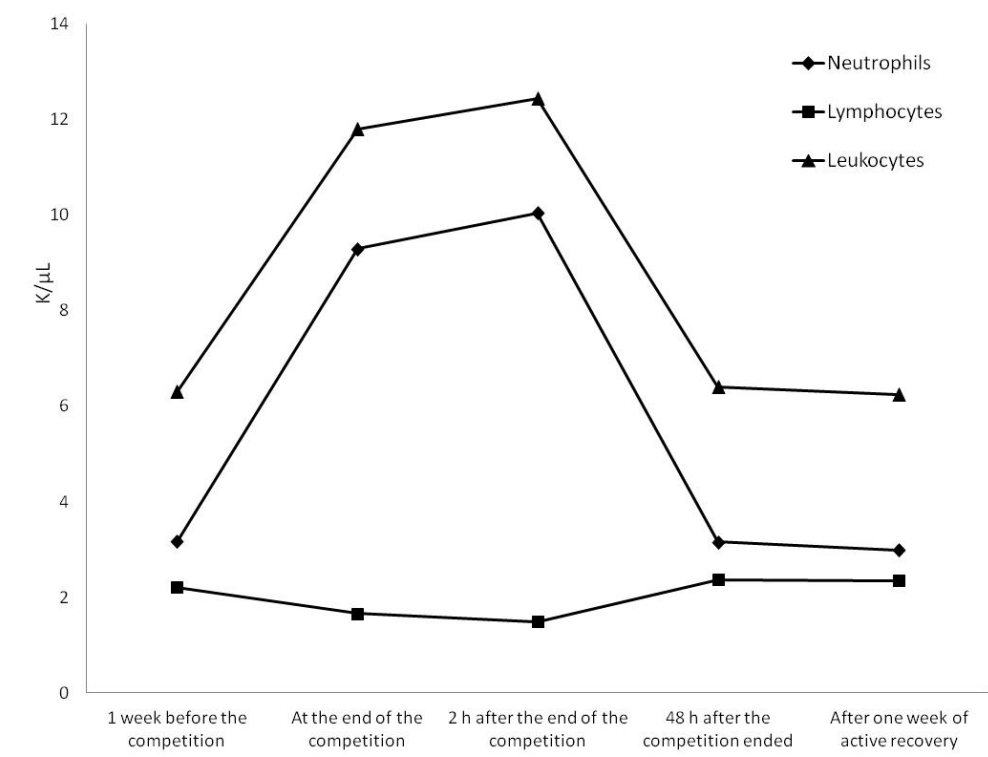

Figure 1

Changes in concentrations of leukocytes, neutrophils and lymphocytes in different evaluation stages.

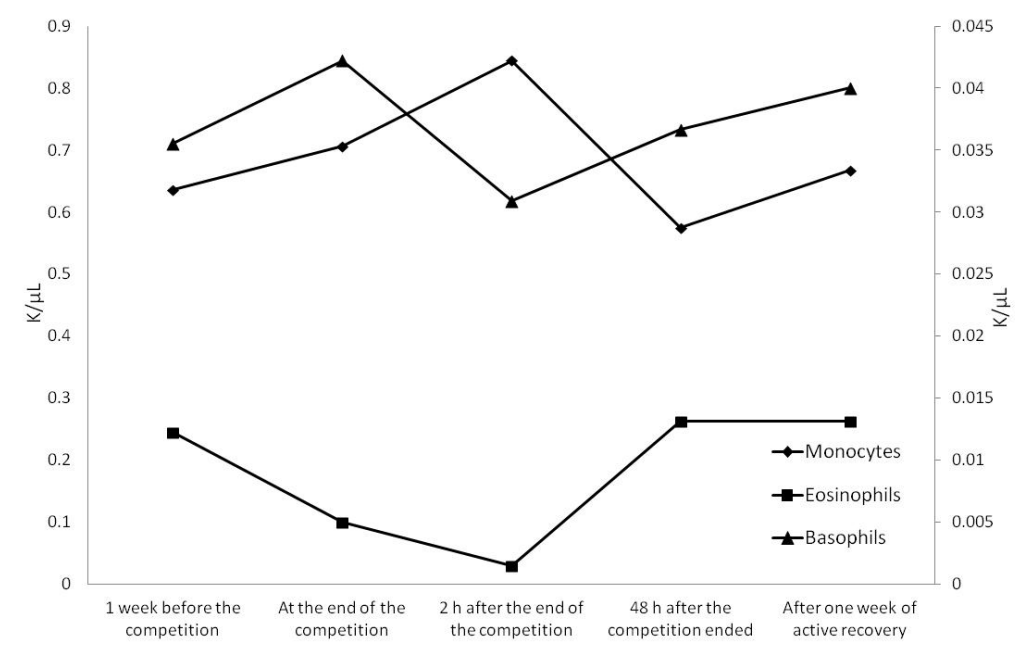

Figure 2

Changes in concentrations of monocytes, eosinophils and basophils at different evaluation stages. The values of the right axis correspond to basophils. 


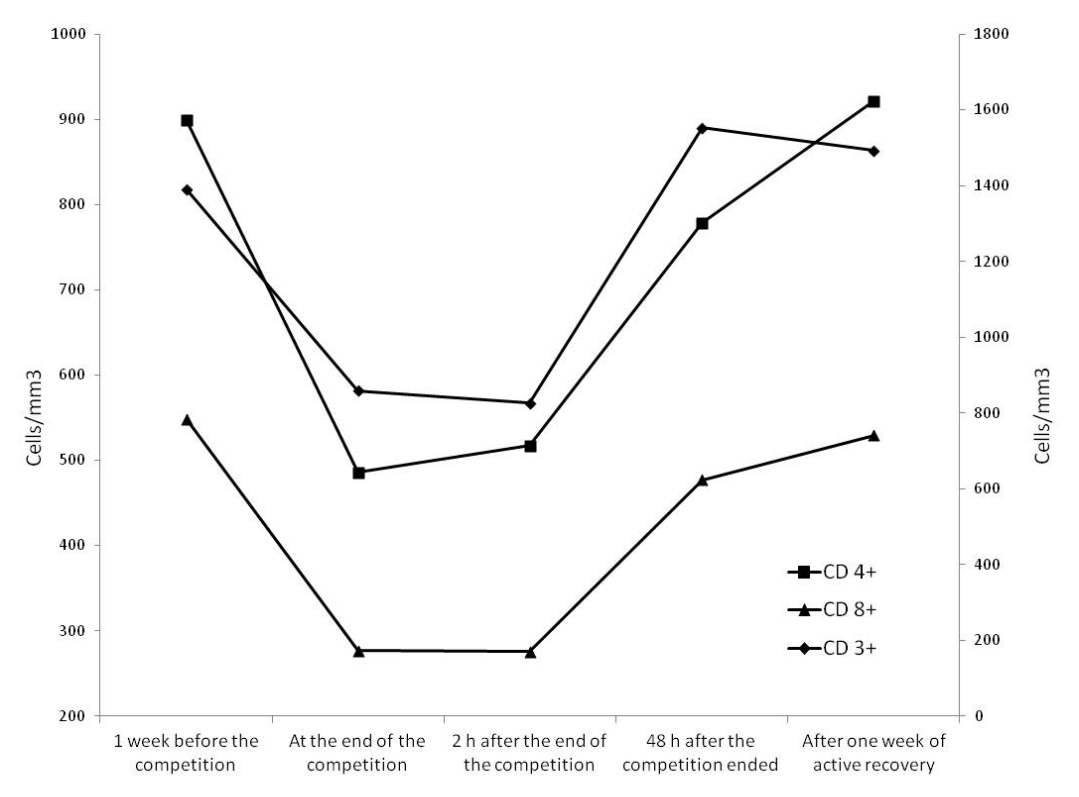

Figure 3

Changes in concentrations of $\mathrm{CD} 3+, \mathrm{CD} 8+$ and $\mathrm{CD} 4+$ at different evaluation stages. The values of the right axis correspond to $C D 3+$.

Leukocytosis in athletes caused by competition is transient and its magnitude is directly related to the intensity of the effort (more pronounced in response to maximal exercise), and inversely related with the level of fitness (Gleeson, 2007; Tossige-Gomes et al., 2014). Leukocytosis especially affects neutrophils, but also monocytes and NK cells (Simpson et al., 2006). There is a possibility that this transient decrease reflects an "open window" in which the defense mechanism is reduced and the risk of infection increases (Kakanis et al., 2010; Wolach et al., 2005).

Neutrophils are the most abundant leukocytes in the bloodstream and are the first responders in viral and bacterial infections. Multiple factors are related to neutrophil behavior and to the immune response during exercise. They act as neuroendocrine mediators, corticosteroid releasing factors, interleukin producers, and are associated with oxidationreduction processes that produce free radicals.
Several studies have shown that most components of circulating leukocytes, mainly neutrophils, increase dramatically during exercise (de Moura et al., 2012; Gavrieli et al., 2008; Kakanis et al., 2010). The magnitude of this increase is related to its duration and intensity. Leukocyte traffic reflects the accumulation of neutrophils in the blood stream and this could be related to hemodynamic changes such as an increase in cardiac function, hyperthermia, and it can reflect changes in circulating stress hormones, mainly epinephrine and cortisol secreted during exercise. A low leukocyte count is generally normal in athletes. During long and high volume training periods, it can be associated with long term suppression in the number of these cells, which can remain low for weeks. This can be attributed to migration of leukocytes from the bloodstream to a site of possible damage to skeletal muscle (Wolach, 2012).

The increase in lymphocytes during 
strenuous exercise followed by a sudden drop or lymphopenia during the recovery phase is reported primarily for the subpopulations CD3+, CD4+, CD8+ and CD56+ (Kakanis et al., 2010; Scharhag, 2005; Simpson et al., 2006). This mobilization is dependent on the expression of activation and adhesion molecules on the cell surface of lymphocytes, for recognition and binding to endothelial cells, facilitating subsequent extravasation and migration into tissues or lymphoid organs. Cordova et al. (2010) reported an increase in CD3+ immediately after vigorous exercise and a decrease at $30 \mathrm{~min}$ of recovery; therefore, it is assumed that the intensity, type of activity and sports level of athletes also influence the behavior of the immune response.

In accordance with previous studies, it was observed that leukocytes, neutrophils and monocytes in athletes increased as a result of the effort of competition and recovered one week later; however, lymphocytes decreased at the end of the competition and were restored a week later. Our results allowed us to observe how these white cells recovered to normal values at 48 hours. Such a condition is associated with inflammatory processes started in response to tissue damage, which from a clinical standpoint trigger all the symptoms of inflammation. This response is characterized by extravasation of fluid, plasma proteins and leukocytes into damaged tissue. These events take place within a few hours after the injury and are linked to the recruitment of leukocytes into the damaged area (Gleeson, 2007; Scharhag, 2005).

Another important finding of the study is the inverse correlation observed between eosinophils and heart rate variability, which is interesting especially considering that these cells are rarely mentioned in other research. Eosinophils have a key role in response to pathogens mainly associated with environmental factors; therefore, an increase would indicate a higher incidence of respiratory diseases in athletes (Bermon, 2007; Morgado et al., 2012).

The correlation between lymphocytes and rMSSD can be considered an indicator that the autonomic nervous system is directly related to the immune system and maintains balance or body homeostasis. At the end of the competition, there is a predominance of the sympathetic system, which induces the release of noradrenalin and stimulation of chromaffin cells of the adrenal glands to secrete adrenaline. This exerts a direct influence on immune cells with receptors for these hormones. Conversely, in a situation of rest, a predominance of the parasympathetic system is observed. Basophils and eosinophils are found in baseline values as well as MRR, SDNN, pNN50 and $\mathrm{rMSSD}$, variables that provide information on the general health status of the individual. We can consider HRV as a useful, noninvasive tool that indicates the function of the autonomic nervous system, providing information about the risk of disease of the immune system (Hellard et al., 2011).

Based on the results obtained in this study, we conclude that there is a correlation between heart rate variability and the level of some of the cells of the immune response resulting from a strenuous effort during triathlon competition and its recovery process. When normal conditions of the immune response are affected through strenuous exercise, this indicates that the individual is susceptible to disease, which can adversely affect athletic performance. Therefore, we believe that through an invasive method such as the analysis of the immune response by blood samples, and HRV as a noninvasive method, the action of the autonomic nervous system can be observed, either during strenuous exercise or during recovery of the athlete, making them useful as a training control method.

\section{References}

Bermon S. Airway inflammation and upper respiratory tract infection in athletes: is there a link? Exerc Immunol Rev, 2007; 13: 6-14

Bricout VA, Dechenaud S, Favre-Juvin A. Analyses of heart rate variability in young soccer players: the effects of sport activity. Auton Neurosci-Basic, 2010; 154: 112-6 
Buchheit M, Voss SC, Nybo L, Mohr M, Racinais S. Physiological and performance adaptations to an inseason soccer camp in the heat: associations with heart rate and heart rate variability. Scand J Med Sci Spor, 2011; 21: 477-85

Córdova A, Sureda A, Tur JA, Pons A. Immune response to exercise in elite sportsmen during the competitive season. J Physiol Biochem, 2010; 66: 1-6

De Moura NR, Cury-Boaventura MF, Santos VC, Levada-Pires AC, Bortolon J, Fiamoncini J, Pithon-Curi TC, Rui C \& Hatanaka E. Inflammatory response and neutrophil functions in players after a futsal match. $J$ Strength Cond Res, 2012; 26: 2507-2514

Gavrieli R, Ashlagi-Amiri T, Eliakim A, Nemet D, Zigel L, Berger-Achituv S, Wolach B. The effect of aerobic exercise on neutrophil functions. Med Sci Sports Exerc, 2008; 40: 1623-1628

Gleeson M. Immune function in sport and exercise. J Appl Physiol, 2007; 103: 693-9

Hellard P, Guimaraes F, Avalos M, Houel N, Hausswirth C, Toussaint JF. Modeling the association between HR variability and illness in elite swimmers. Med Sci Sport Exer, 2011; 43: 1063-70

Kakanis MW, Peake J, Brenu EW, Simmonds M, Gray B. The open window of susceptibility to infection after acute exercise in healthy young male elite athletes. J Sci Med Sport, 2010; 13: 119-137

Levada-Pires AC, Cury-Boaventura MF, Gorjão R, Hirabara SM, Puggina EF, Pellegrinotti IL, Pithon-Curi TC. Induction of lymphocyte death by short- and long-duration triathlon competitions. Med Sci Sport Exer, 2009; 41: 1896-1901

Levada-Pires AC, Cury-Boaventura MF, Gorjão R, Hirabara SM, Puggina EF, Peres CM, Pithon-Curi TC. Neutrophil death induced by a triathlon competition in elite athletes. Med Sci Sport Exer, 2008; 40: 1447-54

Malik M, Bigger J, Camm A. Heart rate variability standards of measurement, physiological interpretation, and clinical use. Eur Heart J, 1996; 17: 354-381

Malm C. Susceptibility to infections in elite athletes: the S-curve. Scand J Med Sci Spor, 2006; 16: 4-6

Marsland AL, Gianaros PJ, Prather AA, Jennings JR, Neumann SA, Manuck SB. Stimulated production of proinflammatory cytokines covaries inversely with heart rate variability. Psychosom Med, 2007; 69: 709-16

Mazon J, Gastaldi A, Di Sacco T, Cozza I, Dutra S, Souza H. Effects of training periodization on cardiac autonomic modulation and endogenous stress markers in volleyball players. Scand J Med Sci Spor, 2013; 23: 114-120

Morgado JM, Rama L, Silva I, de Jesus Inácio M, Henriques A, Laranjeira P, Teixeira AM. Cytokine production by monocytes, neutrophils, and dendritic cells is hampered by long-term intensive training in elite swimmers. Eur J Appl Physiol, 2012; 112: 471-82

Nieman DC. Marathon training and immune function. Sports Med, 2007; 37: 412-415

Nieman DC. Clinical implications of exercise immunology. J Sport Health Sci, 2011; 1: 12-17

Park C, Park T. Changes of immunological markers in elite and amateur triathletes: original research article. Int Sportmed J, 2008; 9: 116-130

Plews DJ, Laursen PB, Kilding AE, Buchheit M. Evaluating training adaptation with heart-rate measures: a methodological comparison. Int J Sport Physiol, 2013; 8: 688-91

Plews DJ, Laursen PB, Stanley J, Kilding AE, Buchheit M. Training adaptation and heart rate variability in elite endurance athletes: opening the door to effective monitoring. Sports Med, 2013; 43: 773-81

Santos VC, Levada-Pires AC, Alves SR, Pithon-Curi TC, Curi R, Cury-Boaventura MF. Changes in lymphocyte and neutrophil function induced by a marathon race. Cell Biochem Funct, 2013; 31: 237-43

Scharhag J. Does prolonged cycling of moderate intensity affect immune cell function? Commentary. Brit J Sport Med, 2005; 39: 171-177 
Simpson RJ, Florida-James GD, Whyte GP, Guy K. The effects of intensive, moderate and downhill treadmill running on human blood lymphocytes expressing the adhesion/activation molecules CD54 (ICAM-1), CD18 (beta2 integrin) and CD53. Eur J Appl Physiol, 2006; 97: 109-21

Tossige-Gomes R, Ottone VO, Oliveira PN, Viana DJS, Araújo TL, Gripp FJ, Rocha-Vieira E. Leukocytosis, muscle damage and increased lymphocyte proliferative response after an adventure sprint race. Braz J Med Biol Res, 2014; 47: 492-498

Wolach B. Exercise and the immune system-Focusing on the effect of exercise on neutrophil functions. INTECH Open Access Publisher, 145-159; 2012

Wolach B, Gavrieli R, Ben-Dror SG, Zigel L, Eliakim A, Falk B. Transient decrease of neutrophil chemotaxis following aerobic exercise. Med Sci Sport Exer, 2005; 37: 949-954

\section{Corresponding author:}

\section{Blanca Rangel-Colmenero}

Ave. Universidad S/N

Ciudad Universitaria, Facultad de Organización Deportiva

San Nicolás de los Garza, Nuevo León, México, C.P. 66451

Phone: +521 13404450 ext. 7633, 7634

E-mail: blanca.rangelc@uanl.mx 